\title{
On the improvement of Fickett's theorem on bounded sets
}

\author{
Soon-Mo Jung ${ }^{1}$, Jaiok Roh ${ }^{2 *}$ (D) and Dae-Jeong Yang ${ }^{3}$
}

${ }^{\text {*Correspondence: }}$

joroh@hallym.ac.kr

${ }^{2}$ Ilsong College of Liberal Arts, Hallym University, Chuncheon

24252, Republic of Korea

Full list of author information is

available at the end of the article

\begin{abstract}
Fickett proved the stability of isometries on bounded subsets of $\mathbb{R}^{n}$ for $n \geq 2$. Jung then improved Fickett's theorem for $n \geq 3$. In this paper, we improve Fickett's theorem for $n=2$ and improve Jung's result for $n=3$, by employing a fundamental analytic method, as it can be used to explain mathematically many practical engineering problems.
\end{abstract}

MSC: Primary 46C99; secondary 39B82; 39B62; 46B04

Keywords: Fickett's theorem; Hyers-Ulam stability; $\varepsilon$-isometry; Isometry

\section{Introduction}

In 1982 Fickett proved the following stability of isometries:

Fickett's Theorem ([4]) For a fixed integer $n \geq 2$, let $D$ be a bounded subset of the Euclidean space $\mathbb{R}^{n}$ and let $\varepsilon>0$ be given. If a function $f: D \rightarrow \mathbb{R}^{n}$ satisfies the inequality

$$
|\|f(x)-f(y)\|-\|x-y\|| \leq \varepsilon
$$

for all $x, y \in D$, then there exists an isometry $U: D \rightarrow \mathbb{R}^{n}$ such that

$$
\|f(x)-U(x)\| \leq 27 \varepsilon^{1 / 2^{n}}
$$

for each $x \in D$.

For any sufficiently small $\varepsilon>0$, one can see that the upper bound of inequality (1.1) can be very large in comparison to $\varepsilon$. This was the motivation for this paper and we will focus on $n=2,3$ as this theorem can be very useful for solving physics problems.

Some mathematicians have tried to improve Fickett's theorem in various ways. Alestalo et al. [1] and Väisälä [13] significantly improved Fickett's result by proving the Hyers-Ulam stability of isometries defined on bounded subsets of $\mathbb{R}^{n}$. Very recently, Jung [8] improved the results of the previous two papers $[1,13]$ significantly in the case of $n \geq 3$. In this paper, we will improve Fickett's results when $n=2$. Moreover, by improving the results of Jung [8] it will also be improved when $n=3$. The reason for focusing on this problem in $\mathbb{R}^{2}$ and

(c) The Author(s) 2022. This article is licensed under a Creative Commons Attribution 4.0 International License, which permits use, sharing, adaptation, distribution and reproduction in any medium or format, as long as you give appropriate credit to the original author(s) and the source, provide a link to the Creative Commons licence, and indicate if changes were made. The images or other third party material in this article are included in the article's Creative Commons licence, unless indicated otherwise in a credit line to the material. If material is not included in the article's Creative Commons licence and your intended use is not permitted by statutory regulation or exceeds the permitted use, you will need to obtain permission directly from the copyright holder. To view a copy of this licence, visit http://creativecommons.org/licenses/by/4.0/. 
$\mathbb{R}^{3}$ is that it can be used to explain mathematically many practical engineering problems that occur in 2-dimensional or 3-dimensional Euclidean spaces. For a mathematical tool, we will use a very fundamental analytic method.

In this paper, we use the notation $\mathbb{K}$ to denote either $\mathbb{R}$ or $\mathbb{C}$. Further, $(E,\|\cdot\|)$ and $(F,\|\cdot\|)$ denote the Hilbert spaces over $\mathbb{K}$. If a function $f: E \rightarrow F$ satisfies the condition

$$
\|f(u)-f(v)\|=\|u-v\|
$$

for all $u, v \in E$, then $f$ is called an isometry.

By recalling the definition of Hyers and Ulam [7], for any fixed $\varepsilon \geq 0$, a function $f: E \rightarrow F$ is called an $\varepsilon$-isometry if $f$ satisfies the inequality

$$
|\|f(u)-f(v)\|-\|u-v\|| \leq \varepsilon
$$

for all $u, v \in E$. If there exists a constant $K>0$ such that for any $\varepsilon>0$ and $\varepsilon$-isometry $f: E \rightarrow F$, there is an isometry $U: E \rightarrow F$ that satisfies $\|f(x)-U(x)\| \leq K \varepsilon$ for all $x \in E$, then the functional equation (1.2) is said to have (or satisfy) the Hyers-Ulam stability. To understand this topic more broadly, one can refer to $[2,3,5-7,9-12]$.

\section{Useful lemmas}

An orthogonal matrix $\mathbf{Q}$ is a real square matrix whose columns and rows are orthonormal vectors. In other words, a real square matrix $\mathbf{Q}$ is orthogonal if its transpose is equal to its inverse: $\mathbf{Q}^{\text {tr }}=\mathbf{Q}^{-1}$. As a linear transformation, an orthogonal matrix preserves the inner product of vectors, and therefore acts as an isometry of Euclidean space.

Now, we introduce the familiar theorem called QR decomposition. We omit the proof because it can be proved by using the Gram-Schmidt process.

Lemma 2.1 ( $\mathrm{QR}$ decomposition) Every real square matrix A can be decomposed as $\mathbf{A}=\mathbf{Q R}$, where $\mathbf{Q}$ is an orthogonal matrix and $\mathbf{R}$ is an upper triangular matrix with nonnegative diagonal elements.

The following lemma will be used to almost halve $n^{2}$ unknowns. We will prove it by applying QR decomposition mentioned above. For the proof, one can refer to [8].

Lemma 2.2 Given an integer $n>0$, let $\left\{e_{1}, e_{2}, \ldots, e_{n}\right\}$ be the standard basis for the $n$ dimensional Euclidean space $\mathbb{R}^{n}$, where each $e_{i}$ is written in a column vector, let $D$ be a subset of $\mathbb{R}^{n}$ that satisfies $\left\{e_{1}, e_{2}, \ldots, e_{n}\right\} \subset D$, and let $f: D \rightarrow \mathbb{R}^{n}$ be a function. Then, there exist an orthogonal matrix $\mathbf{Q}$ and real numbers $e_{i j}^{\prime}$ for $i, j \in\{1,2, \ldots, n\}$ with $i \geq j$ such that

$$
\mathbf{Q}^{\operatorname{tr}} f\left(e_{i}\right)=\left(e_{i 1}^{\prime}, e_{i 2}^{\prime}, \ldots, e_{i i}^{\prime}, 0, \ldots, 0\right)^{\operatorname{tr}}
$$

for every $i \in\{1,2, \ldots, n\}$. In particular, $e_{i i}^{\prime} \geq 0$ for all $i \in\{1,2, \ldots, n\}$.

\section{Main theorems}

From now on, we use the symbol $\|x\|$ to denote the norm of $x$ defined by $\|x\|:=\sqrt{x_{1}^{2}+x_{2}^{2}}$ or $\|x\|:=\sqrt{x_{1}^{2}+x_{2}^{2}+x_{3}^{2}}$ for each $x=\left(x_{1}, x_{2}\right) \in \mathbb{R}^{2}$ or $x=\left(x_{1}, x_{2}, x_{3}\right) \in \mathbb{R}^{3}$, respectively. 


\subsection{On bounded subsets of $\mathbb{R}^{2}$}

To prove the next lemma we will use Lemma 2.2 to almost halve the number of unknowns to consider.

In the following lemma, the parameters $c_{i j}$ are assumed to be positive real numbers, while they were assumed to be positive integers in [8].

Lemma 3.1 Let $\left\{e_{1}, e_{2}\right\}$ be the standard basis for the 2-dimensional Euclidean space $\mathbb{R}^{2}$, let $D$ be a subset of $\mathbb{R}^{2}$ that includes $0, e_{1}$, and $e_{2}$, and let $f: D \rightarrow \mathbb{R}^{2}$ be a function that satisfies $f(0)=0$ and the inequality (1.3) for all $x, y \in\left\{0, e_{1}, e_{2}\right\}$ and for some constant $\varepsilon$ with $0<\varepsilon \leq \frac{1}{13}$. In view of Lemma 2.2 , it can be assumed that $f\left(e_{1}\right)=\left(e_{11}^{\prime}, 0\right)$ and $f\left(e_{2}\right)=\left(e_{21}^{\prime}, e_{22}^{\prime}\right)$, where $e_{11}^{\prime} \geq 0$ and $e_{22}^{\prime} \geq 0$. Then, there exist real numbers $c_{i j}>0, i, j \in\{1,2\}$ with $j \leq i$, such that

$$
\begin{cases}-c_{i j} \varepsilon \leq e_{i j}^{\prime} \leq c_{i j} \varepsilon & (\text { for } i>j) \\ 1-c_{i i} \varepsilon \leq e_{i i}^{\prime} \leq 1+\varepsilon & (\text { for } i=j)\end{cases}
$$

In particular, $c_{11}=1.0000000, c_{21} \approx 3.7403981$, and $c_{22} \approx 1.5978326$ may be selected.

Proof In view of (1.3) and $f(0)=0$, we have

$$
||\left|f\left(e_{1}\right)\left\|-1|\leq \varepsilon, \quad||| f\left(e_{2}\right)\right\|-1\right| \leq \varepsilon, \quad|| f\left(e_{1}\right)-f\left(e_{2}\right) \|-\sqrt{2} \mid \leq \varepsilon
$$

for any $\varepsilon$ with $0<\varepsilon \leq \frac{1}{13}$. Hence, it follows from these inequalities that

$$
\begin{aligned}
& 1-\varepsilon \leq e_{11}^{\prime} \leq 1+\varepsilon, \\
& (1-\varepsilon)^{2} \leq e_{21}^{\prime 2}+e_{22}^{\prime 2} \leq(1+\varepsilon)^{2}, \\
& (\sqrt{2}-\varepsilon)^{2} \leq\left(e_{11}^{\prime}-e_{21}^{\prime}\right)^{2}+e_{22}^{\prime 2} \leq(\sqrt{2}+\varepsilon)^{2} .
\end{aligned}
$$

It follows from (3.4) that

$$
(\sqrt{2}-\varepsilon)^{2}-e_{11}^{\prime 2}-\left(e_{21}^{\prime 2}+e_{22}^{\prime 2}\right) \leq-2 e_{11}^{\prime} e_{21}^{\prime} \leq(\sqrt{2}+\varepsilon)^{2}-e_{11}^{\prime 2}-\left(e_{21}^{\prime 2}+e_{22}^{\prime 2}\right)
$$

We recall that $0<\varepsilon \leq \frac{1}{13}$. By (3.2) and (3.3), we obtain

$$
-\frac{26+13 \sqrt{2}}{12} \varepsilon \leq \frac{-(4+2 \sqrt{2}) \varepsilon+\varepsilon^{2}}{2(1-\varepsilon)} \leq e_{21}^{\prime} \leq \frac{(4+2 \sqrt{2}) \varepsilon+\varepsilon^{2}}{2(1-\varepsilon)} \leq \frac{53+26 \sqrt{2}}{24} \varepsilon .
$$

Now, we select the smallest possible positive real number $c_{21}$ that satisfies

$$
-c_{21} \varepsilon \leq-\frac{26+13 \sqrt{2}}{12} \varepsilon \leq e_{21}^{\prime} \leq \frac{53+26 \sqrt{2}}{24} \varepsilon \leq c_{21} \varepsilon .
$$

Hence, we may choose

$$
c_{21}=\frac{53+26 \sqrt{2}}{24} \approx 3.7403981
$$

as the smallest possible positive number that satisfies the last inequality. 
Furthermore, using our assumption that $0<\varepsilon \leq \frac{1}{13}$, it follows from (3.1) with $i=2$ and $j=1,(3.3)$, and (3.5) that

$$
1-2 \varepsilon-\frac{3585+2756 \sqrt{2}}{576} \varepsilon^{2}=1-2 \varepsilon+\left(1-c_{21}^{2}\right) \varepsilon^{2} \leq e_{22}^{\prime 2} \leq(1+\varepsilon)^{2} .
$$

By solving the following inequality, where $c_{22}$ is unknown,

$$
\left(1-c_{22} \varepsilon\right)^{2} \leq 1-2 \varepsilon-\frac{3585+2756 \sqrt{2}}{576} \varepsilon^{2}
$$

we obtain

$$
\begin{aligned}
& \frac{1}{\varepsilon}\left(1-\sqrt{1-2 \varepsilon-\frac{3585+2756 \sqrt{2}}{576} \varepsilon^{2}}\right) \\
& \leq c_{22} \leq \frac{1}{\varepsilon}\left(1+\sqrt{1-2 \varepsilon-\frac{3585+2756 \sqrt{2}}{576}} \varepsilon^{2}\right) .
\end{aligned}
$$

Thus, putting $\varepsilon=\frac{1}{13}$ in the lower bound for $c_{22}$ in (3.6), we may select

$$
c_{22}=13-\sqrt{\frac{78,783-2756 \sqrt{2}}{576}} \approx 1.5978326
$$

as the smallest possible positive constant that satisfies the inequality (3.6).

In the following theorem, we set $e_{1}=(1,0)$ and $e_{2}=(0,1)$ such that $\left\{e_{1}, e_{2}\right\}$ is the standard basis for the 2-dimensional Euclidean space $\mathbb{R}^{2}$. We denote by $B_{d}(0)$ the closed ball of radius $d$ and centered at the origin of $\mathbb{R}^{2}$, i.e., $B_{d}(0)=\left\{x \in \mathbb{R}^{2}:\|x\| \leq d\right\}$.

Theorem 3.2 Let $D$ be a subset of the Euclidean space $\mathbb{R}^{2}$ such that $\left\{0, e_{1}, e_{2}\right\} \subset D \subset B_{d}(0)$ for some $d \geq 1$ and let $f: D \rightarrow \mathbb{R}^{2}$ be a function that satisfies $f(0)=0$ and the inequality (1.3) for all $x, y \in D$ and for some constant $\varepsilon$ with $0<\varepsilon \leq \frac{1}{13}$. Then, there exists an isometry $U: D \rightarrow \mathbb{R}^{2}$ such that

$$
\|f(x)-U(x)\| \leq(8 d+4) \varepsilon
$$

for all $x \in D$.

Proof Due to Lemma 2.2, we may assume that $f\left(e_{1}\right)=\left(e_{11}^{\prime}, 0\right)$ and $f\left(e_{2}\right)=\left(e_{21}^{\prime}, e_{22}^{\prime}\right)$ with $e_{11}^{\prime} \geq 0$ and $e_{22}^{\prime} \geq 0$. For every point $x=\left(x_{1}, x_{2}\right) \in D$, let $f(x)=x^{\prime}=\left(x_{1}^{\prime}, x_{2}^{\prime}\right)$. Then, by (1.3), we have

$$
\begin{aligned}
& \left|\sqrt{x_{1}^{\prime 2}+x_{2}^{\prime 2}}-\sqrt{x_{1}^{2}+x_{2}^{2}}\right| \leq \varepsilon, \\
& \left|\sqrt{\left(x_{1}^{\prime}-e_{11}^{\prime}\right)^{2}+x_{2}^{\prime 2}}-\sqrt{\left(x_{1}-1\right)^{2}+x_{2}^{2}}\right| \leq \varepsilon, \\
& \left|\sqrt{\left(x_{1}^{\prime}-e_{21}^{\prime}\right)^{2}+\left(x_{2}^{\prime}-e_{22}^{\prime}\right)^{2}}-\sqrt{x_{1}^{2}+\left(x_{2}-1\right)^{2}}\right| \leq \varepsilon .
\end{aligned}
$$


According to (3.9), we obtain

$$
\begin{aligned}
& \left|\left(x_{1}^{\prime 2}+x_{2}^{\prime 2}\right)-\left(x_{1}^{2}+x_{2}^{2}\right)\right| \\
& \quad=\left|\sqrt{x_{1}^{\prime 2}+x_{2}^{\prime 2}}-\sqrt{x_{1}^{2}+x_{2}^{2}}\right|\left|\sqrt{x_{1}^{\prime 2}+x_{2}^{\prime 2}}+\sqrt{x_{1}^{2}+x_{2}^{2}}\right| \\
& \quad \leq \varepsilon(d+\varepsilon+d) \leq\left(2 d+\frac{1}{13}\right) \varepsilon
\end{aligned}
$$

since $\sqrt{x_{1}^{\prime 2}+x_{2}^{\prime 2}}=\|f(x)\| \leq\|x\|+\varepsilon \leq d+\varepsilon, \sqrt{x_{1}^{2}+x_{2}^{2}} \leq d$ and $0<\varepsilon \leq \frac{1}{13}$. Similarly, by (3.10), we obtain

$$
\left|\left(\left(x_{1}^{\prime}-e_{11}^{\prime}\right)^{2}+x_{2}^{\prime 2}\right)-\left(\left(x_{1}-1\right)^{2}+x_{2}^{2}\right)\right| \leq\left(2 d+\frac{27}{13}\right) \varepsilon
$$

since $\sqrt{\left(x_{1}^{\prime}-e_{11}^{\prime}\right)^{2}+x_{2}^{\prime 2}}=\left\|f(x)-f\left(e_{1}\right)\right\| \leq\left\|x-e_{1}\right\|+\varepsilon \leq d+1+\varepsilon, \sqrt{\left(x_{1}-1\right)^{2}+x_{2}^{2}}=\| x-$ $e_{1} \| \leq d+1$ and $0<\varepsilon \leq \frac{1}{13}$. Analogously, in view of (3.11), we have

$$
\left|\left(\left(x_{1}^{\prime}-e_{21}^{\prime}\right)^{2}+\left(x_{2}^{\prime}-e_{22}^{\prime}\right)^{2}\right)-\left(x_{1}^{2}+\left(x_{2}-1\right)^{2}\right)\right| \leq\left(2 d+\frac{27}{13}\right) \varepsilon
$$

since $\sqrt{\left(x_{1}^{\prime}-e_{21}^{\prime}\right)^{2}+\left(x_{2}^{\prime}-e_{22}^{\prime}\right)^{2}}=\left\|f(x)-f\left(e_{2}\right)\right\| \leq\left\|x-e_{2}\right\|+\varepsilon \leq d+1+\varepsilon$ and $0<\varepsilon \leq \frac{1}{13}$. It now follows from (3.13) that

$$
\left|\left(x_{1}^{\prime 2}+x_{2}^{\prime 2}\right)-\left(x_{1}^{2}+x_{2}^{2}\right)-2 e_{11}^{\prime} x_{1}^{\prime}+2 x_{1}+e_{11}^{\prime 2}-1\right| \leq\left(2 d+\frac{27}{13}\right) \varepsilon
$$

Using (3.12), we obtain

$$
-\left(4 d+\frac{28}{13}\right) \varepsilon \leq-2 e_{11}^{\prime} x_{1}^{\prime}+2 x_{1}+e_{11}^{\prime 2}-1 \leq\left(4 d+\frac{28}{13}\right) \varepsilon
$$

Similarly, using (3.14), we obtain

$$
\left|\left(x_{1}^{\prime 2}+x_{2}^{\prime 2}\right)-\left(x_{1}^{2}+x_{2}^{2}\right)-2 e_{21}^{\prime} x_{1}^{\prime}-2 e_{22}^{\prime} x_{2}^{\prime}+2 x_{2}+e_{21}^{\prime 2}+e_{22}^{\prime 2}-1\right| \leq\left(2 d+\frac{27}{13}\right) \varepsilon
$$

Using (3.12) again, we obtain

$$
-\left(4 d+\frac{28}{13}\right) \varepsilon \leq-2 e_{21}^{\prime} x_{1}^{\prime}-2 e_{22}^{\prime} x_{2}^{\prime}+2 x_{2}+e_{21}^{\prime 2}+e_{22}^{\prime 2}-1 \leq\left(4 d+\frac{28}{13}\right) \varepsilon
$$

Moreover, put $x=e_{1}$ and $y=0$ in (1.3) and use (3.1) to obtain

$$
\left|e_{11}^{\prime}-1\right| \leq \varepsilon \quad \text { and } \quad\left|e_{11}^{\prime 2}-1\right|=\left|e_{11}^{\prime}-1\right|\left|e_{11}^{\prime}+1\right| \leq \frac{27}{13} \varepsilon
$$

Hence, it follows from (3.15) that

$$
\left|x_{1}-x_{1}^{\prime}\right| \leq\left(3 d+\frac{57}{26}\right) \varepsilon
$$


since $\max \left\{\left|x_{1}^{\prime}\right|,\left|x_{2}^{\prime}\right|\right\} \leq\|f(x)\| \leq\|x\|+\varepsilon \leq d+\frac{1}{13}$ and

$$
-2 e_{11}^{\prime} x_{1}^{\prime}+2 x_{1}+e_{11}^{\prime 2}-1=2\left(1-e_{11}^{\prime}\right) x_{1}^{\prime}+2\left(x_{1}-x_{1}^{\prime}\right)+e_{11}^{\prime 2}-1 .
$$

On the other hand, it follows from (3.1) that

$$
\left|2 e_{21}^{\prime} x_{1}^{\prime}\right| \leq 2 c_{21}\left|x_{1}^{\prime}\right| \varepsilon \leq 2 c_{21}\left(d+\frac{1}{13}\right) \varepsilon
$$

Due to (3.1), we obtain

$$
-\varepsilon \leq 1-e_{22}^{\prime} \leq c_{22} \varepsilon
$$

By (3.16) together with (3.3), we obtain

$$
-\left(\left(2+c_{21}\right) d+\frac{55+2 c_{21}}{26}\right) \varepsilon \leq x_{2}-e_{22}^{\prime} x_{2}^{\prime} \leq\left(\left(2+c_{21}\right) d+\frac{27+c_{21}}{13}\right) \varepsilon,
$$

or since $x_{2}-e_{22}^{\prime} x_{2}^{\prime}=\left(x_{2}-x_{2}^{\prime}\right)+\left(1-e_{22}^{\prime}\right) x_{2}^{\prime}$,

$$
\begin{aligned}
& -\left(\left(2+c_{21}+c_{22}\right) d+\frac{55+2 c_{21}+2 c_{22}}{26}\right) \varepsilon \\
& \quad \leq x_{2}-x_{2}^{\prime} \leq\left(\left(2+c_{21}+c_{22}\right) d+\frac{27+c_{21}+c_{22}}{13}\right) \varepsilon .
\end{aligned}
$$

Therefore, we have

$$
\left|x_{2}-x_{2}^{\prime}\right| \leq\left(\left(2+c_{21}+c_{22}\right) d+\frac{55+2 c_{21}+2 c_{22}}{26}\right) \varepsilon .
$$

Finally, we define an isometry $U: D \rightarrow \mathbb{R}^{2}$ by $U(x)=x=\left(x_{1}, x_{2}\right)$. It then follows from (3.17), (3.18) and Lemma 3.1 that

$$
\begin{aligned}
\|f(x)-U(x)\| & =\|f(x)-x\|=\left\|\left(x_{1}^{\prime}-x_{1}, x_{2}^{\prime}-x_{2}\right)\right\| \\
& =\sqrt{\left(x_{1}^{\prime}-x_{1}\right)^{2}+\left(x_{2}^{\prime}-x_{2}\right)^{2}} \\
& \leq(8 d+4) \varepsilon,
\end{aligned}
$$

which completes our proof.

By $c_{i j}$ in Lemma 3.1, (3.17) and (3.18), we can express the upper bound of inequality (3.8) more precisely using rational numbers as:

$$
\begin{aligned}
\|f(x)-U(x)\| & =\sqrt{62.8496299 d^{2}+50.2268490 d+11.1869791} \varepsilon \\
& \leq \sqrt{62.8496299 d^{2}+51.0138281 d+10.4000000} \varepsilon \\
& \leq(7.9277759 d+3.2249031) \varepsilon .
\end{aligned}
$$




\subsection{On bounded subsets of $\mathbb{R}^{3}$}

Lemma 3.1 can now be extended without difficulty to the case of the 3-dimensional Euclidean space. It is evident that there exist positive real numbers $c_{i j}, i, j \in\{1,2,3\}$ with $j \leq i$ that satisfy the conditions in (3.1) whenever the function $f$ satisfies $f(0)=0$ and the inequality (1.3) for all $x, y \in\left\{0, e_{1}, e_{2}, e_{3}\right\}$.

In the following lemma, the parameters $c_{i j}$ are assumed to be positive real numbers, while they were assumed to be positive integers in [8].

Lemma 3.3 Let $\left\{e_{1}, e_{2}, e_{3}\right\}$ be the standard basis for the 3-dimensional Euclidean space $\mathbb{R}^{3}$, let $D$ be a subset of $\mathbb{R}^{3}$ that satisfies $\left\{0, e_{1}, e_{2}, e_{3}\right\} \subset D$, and let $f: D \rightarrow \mathbb{R}^{3}$ be a function that satisfies $f(0)=0$ and the inequality (1.3) for all $x, y \in\left\{0, e_{1}, e_{2}, e_{3}\right\}$ and for some constant $\varepsilon$ with $0<\varepsilon \leq \frac{1}{13}$. By Lemma 2.2, it can be assumed that $f\left(e_{1}\right)=\left(e_{11}^{\prime}, 0,0\right), f\left(e_{2}\right)=\left(e_{21}^{\prime}, e_{22}^{\prime}, 0\right)$, and $f\left(e_{3}\right)=\left(e_{31}^{\prime}, e_{32}^{\prime}, e_{33}^{\prime}\right)$, where $e_{11}^{\prime} \geq 0, e_{22}^{\prime} \geq 0$, and $e_{33}^{\prime} \geq 0$. Then, there exist positive real numbers $c_{i j}, i, j \in\{1,2,3\}$ with $j \leq i$ that satisfy the inequalities in (3.1). In particular, $c_{11}=$ $1.0000000, c_{21} \approx 3.7403981, c_{22} \approx 1.5978326, c_{31} \approx 3.7403981, c_{32} \approx 5.1635231$, and $c_{33} \approx$ 2.8340052 may be selected.

Proof In view of $(1.3)$ and $f(0)=0$, we have

$$
\begin{aligned}
& ||\left|f\left(e_{1}\right)\left\|-1|\leq \varepsilon, \quad||| f\left(e_{2}\right)\right\|-1\right| \leq \varepsilon, \quad||\left|f\left(e_{3}\right) \|-1\right| \leq \varepsilon, \\
& ||\left|f\left(e_{1}\right)-f\left(e_{2}\right)\left\|-\sqrt{2}|\leq \varepsilon, \quad|\left|f\left(e_{2}\right)-f\left(e_{3}\right) \|-\sqrt{2}\right| \leq \varepsilon,\right.\right. \\
& ||\left|f\left(e_{3}\right)-f\left(e_{1}\right) \|-\sqrt{2}\right| \leq \varepsilon
\end{aligned}
$$

for any $\varepsilon$ with $0<\varepsilon \leq \frac{1}{13}$. Therefore, from the inequalities above, we obtain the following inequalities along with (3.2), (3.3) and (3.4):

$$
\begin{aligned}
& (1-\varepsilon)^{2} \leq e_{31}^{\prime 2}+e_{32}^{\prime 2}+e_{33}^{\prime 2} \leq(1+\varepsilon)^{2} \\
& (\sqrt{2}-\varepsilon)^{2} \leq\left(e_{21}^{\prime}-e_{31}^{\prime}\right)^{2}+\left(e_{22}^{\prime}-e_{32}^{\prime}\right)^{2}+e_{33}^{\prime 2} \leq(\sqrt{2}+\varepsilon)^{2} \\
& (\sqrt{2}-\varepsilon)^{2} \leq\left(e_{31}^{\prime}-e_{11}^{\prime}\right)^{2}+e_{32}^{\prime 2}+e_{33}^{\prime 2} \leq(\sqrt{2}+\varepsilon)^{2}
\end{aligned}
$$

Moreover, using (3.21), we have

$$
(\sqrt{2}-\varepsilon)^{2}-e_{11}^{\prime 2}-\left(e_{31}^{\prime 2}+e_{32}^{\prime 2}+e_{33}^{\prime 2}\right) \leq-2 e_{11}^{\prime} e_{31}^{\prime} \leq(\sqrt{2}+\varepsilon)^{2}-e_{11}^{\prime 2}-\left(e_{31}^{\prime 2}+e_{32}^{\prime 2}+e_{33}^{\prime 2}\right)
$$

In view of (3.2) and (3.19), we obtain

$$
\frac{-(4+2 \sqrt{2}) \varepsilon+\varepsilon^{2}}{2(1-\varepsilon)} \leq e_{31}^{\prime} \leq \frac{(4+2 \sqrt{2}) \varepsilon+\varepsilon^{2}}{2(1-\varepsilon)}
$$

and we solve the following inequalities

$$
-c_{31} \varepsilon \leq \frac{-(4+2 \sqrt{2}) \varepsilon+\varepsilon^{2}}{2(1-\varepsilon)} \text { and } \frac{(4+2 \sqrt{2}) \varepsilon+\varepsilon^{2}}{2(1-\varepsilon)} \leq c_{31} \varepsilon
$$


and we find

$$
c_{31}=\frac{53+26 \sqrt{2}}{24} \approx 3.7403981
$$

as the smallest possible positive real number that satisfies the preceding inequalities.

By (3.20), we obtain

$$
\begin{aligned}
2- & 2 \sqrt{2} \varepsilon+\varepsilon^{2}-\left(e_{21}^{\prime 2}+e_{22}^{\prime 2}\right)-\left(e_{31}^{\prime 2}+e_{32}^{\prime 2}+e_{33}^{\prime 2}\right) \\
& \leq-2 e_{21}^{\prime} e_{31}^{\prime}-2 e_{22}^{\prime} e_{32}^{\prime} \leq 2+2 \sqrt{2} \varepsilon+\varepsilon^{2}-\left(e_{21}^{\prime 2}+e_{22}^{\prime 2}\right)-\left(e_{31}^{\prime 2}+e_{32}^{\prime 2}+e_{33}^{\prime 2}\right) .
\end{aligned}
$$

Moreover, we use (3.3) and (3.19) to obtain

$$
-(4+2 \sqrt{2}) \varepsilon+\varepsilon^{2}-2 e_{21}^{\prime} e_{31}^{\prime} \leq 2 e_{22}^{\prime} e_{32}^{\prime} \leq(4+2 \sqrt{2}) \varepsilon+\varepsilon^{2}-2 e_{21}^{\prime} e_{31}^{\prime}
$$

or one time using (3.1) with $i=2$ and $j=1$ and the next time using (3.1) with $i=3$ and $j=1$,

$$
-(4+2 \sqrt{2}) \varepsilon+\varepsilon^{2}-2 c_{21} c_{31} \varepsilon^{2} \leq 2 e_{22}^{\prime} e_{32}^{\prime} \leq(4+2 \sqrt{2}) \varepsilon+\varepsilon^{2}+2 c_{21} c_{31} \varepsilon^{2} .
$$

Due to (3.1) with $i=j=2$, we have

$$
\frac{-(4+2 \sqrt{2}) \varepsilon+\varepsilon^{2}-2 c_{21} c_{31} \varepsilon^{2}}{2\left(1-c_{22} \varepsilon\right)} \leq e_{32}^{\prime} \leq \frac{(4+2 \sqrt{2}) \varepsilon+\varepsilon^{2}+2 c_{21} c_{31} \varepsilon^{2}}{2\left(1-c_{22} \varepsilon\right)}
$$

and we solve the following inequalities

$$
-c_{32} \varepsilon \leq \frac{-(4+2 \sqrt{2}) \varepsilon+\varepsilon^{2}-2 c_{21} c_{31} \varepsilon^{2}}{2\left(1-c_{22} \varepsilon\right)} \quad \text { and } \quad \frac{(4+2 \sqrt{2}) \varepsilon+\varepsilon^{2}+2 c_{21} c_{31} \varepsilon^{2}}{2\left(1-c_{22} \varepsilon\right)} \leq c_{32} \varepsilon
$$

We use (3.5), (3.7), and (3.22) and put $\varepsilon=\frac{1}{13}$ in the second inequality to obtain

$$
c_{32}=\frac{4+2 \sqrt{2}+\frac{1}{13}\left(1+2 \times 3.7403981^{2}\right)}{2\left(1-\frac{1}{13} \times 1.5978326\right)} \approx 5.1635231
$$

as the smallest possible positive number that satisfies the last inequalities.

Finally, by (3.1) with $i=3$ and $j=1$, (3.1) with $i=3$ and $j=2$, and by (3.19), we have

$$
1-2 \varepsilon-\left(c_{31}^{2}+c_{32}^{2}-1\right) \varepsilon^{2} \leq e_{33}^{\prime 2} \leq(1+\varepsilon)^{2} .
$$

Moreover, we solve the following inequality

$$
\left(1-c_{33} \varepsilon\right)^{2} \leq 1-2 \varepsilon-\left(c_{31}^{2}+c_{32}^{2}-1\right) \varepsilon^{2},
$$

whose solution is given as

$$
\frac{1}{\varepsilon}\left(1-\sqrt{1-2 \varepsilon-\left(c_{31}^{2}+c_{32}^{2}-1\right) \varepsilon^{2}}\right) \leq c_{33} \leq \frac{1}{\varepsilon}\left(1+\sqrt{1-2 \varepsilon-\left(c_{31}^{2}+c_{32}^{2}-1\right) \varepsilon^{2}}\right) .
$$


In addition, we use (3.22) and (3.23) and put $\varepsilon=\frac{1}{13}$ in the lower bound for $c_{33}$ to obtain

$$
c_{33}=13-\sqrt{144-c_{31}^{2}-c_{32}^{2}} \approx 2.8340052
$$

as small a positive real number as possible that satisfies the last inequalities.

In the following theorem, we set $e_{1}=(1,0,0), e_{2}=(0,1,0)$, and $e_{3}=(0,0,1)$ such that $\left\{e_{1}, e_{2}, e_{3}\right\}$ is the standard basis for the 3 -dimensional Euclidean space $\mathbb{R}^{3}$. We denote by $B_{d}(0)$ the closed ball of radius $d$ and centered at the origin of $\mathbb{R}^{3}$, i.e., $B_{d}(0)=\left\{x \in \mathbb{R}^{3}\right.$ : $\|x\| \leq d\}$.

Theorem 3.4 Let $D$ be a subset of the 3-dimensional Euclidean space $\mathbb{R}^{3}$ such that $\left\{0, e_{1}, e_{2}, e_{3}\right\} \subset D \subset B_{d}(0)$ for some $d \geq 1$ and let $f: D \rightarrow \mathbb{R}^{3}$ be a function that satisfies $f(0)=0$ and the inequality (1.3) for all $x, y \in D$ and for some constant $\varepsilon$ with $0<\varepsilon \leq \frac{1}{13}$. Then, there exists an isometry $U: D \rightarrow \mathbb{R}^{3}$ such that

$$
\|f(x)-U(x)\| \leq(16 d+5) \varepsilon
$$

for all $x \in D$.

Proof Considering Lemma 2.2, we can assume that $f\left(e_{1}\right)=\left(e_{11}^{\prime}, 0,0\right), f\left(e_{2}\right)=\left(e_{21}^{\prime}, e_{22}^{\prime}, 0\right)$, and $f\left(e_{3}\right)=\left(e_{31}^{\prime}, e_{32}^{\prime}, e_{33}^{\prime}\right)$, where $e_{11}^{\prime} \geq 0, e_{22}^{\prime} \geq 0$, and $e_{33}^{\prime} \geq 0$.

For any point $x=\left(x_{1}, x_{2}, x_{3}\right)$ of $D$, let $f(x)=x^{\prime}=\left(x_{1}^{\prime}, x_{2}^{\prime}, x_{3}^{\prime}\right)$. It then follows from (1.3) that

$$
\begin{aligned}
& \left|\sqrt{x_{1}^{\prime 2}+x_{2}^{\prime 2}+x_{3}^{\prime 2}}-\sqrt{x_{1}^{2}+x_{2}^{2}+x_{3}^{2}}\right| \leq \varepsilon, \\
& \left|\sqrt{\left(x_{1}^{\prime}-e_{11}^{\prime}\right)^{2}+x_{2}^{\prime 2}+x_{3}^{\prime 2}}-\sqrt{\left(x_{1}-1\right)^{2}+x_{2}^{2}+x_{3}^{2}}\right| \leq \varepsilon, \\
& \left|\sqrt{\left(x_{1}^{\prime}-e_{21}^{\prime}\right)^{2}+\left(x_{2}^{\prime}-e_{22}^{\prime}\right)^{2}+x_{3}^{\prime 2}}-\sqrt{x_{1}^{2}+\left(x_{2}-1\right)^{2}+x_{3}^{2}}\right| \leq \varepsilon, \\
& \left|\sqrt{\left(x_{1}^{\prime}-e_{31}^{\prime}\right)^{2}+\left(x_{2}^{\prime}-e_{32}^{\prime}\right)^{2}+\left(x_{3}^{\prime}-e_{33}^{\prime}\right)^{2}}-\sqrt{x_{1}^{2}+x_{2}^{2}+\left(x_{3}-1\right)^{2}}\right| \leq \varepsilon .
\end{aligned}
$$

It follows from (3.26) that

$$
\begin{aligned}
& \left|\left(x_{1}^{\prime 2}+x_{2}^{\prime 2}+x_{3}^{\prime 2}\right)-\left(x_{1}^{2}+x_{2}^{2}+x_{3}^{2}\right)\right| \\
& \quad=\left|\sqrt{x_{1}^{\prime 2}+x_{2}^{\prime 2}+x_{3}^{\prime 2}}-\sqrt{x_{1}^{2}+x_{2}^{2}+x_{3}^{2}}\right|\left|\sqrt{x_{1}^{\prime 2}+x_{2}^{\prime 2}+x_{3}^{\prime 2}}+\sqrt{x_{1}^{2}+x_{2}^{2}+x_{3}^{2}}\right| \\
& \quad \leq \varepsilon(d+\varepsilon+d) \leq\left(2 d+\frac{1}{13}\right) \varepsilon
\end{aligned}
$$

since $\sqrt{x_{1}^{\prime 2}+x_{2}^{\prime 2}+x_{3}^{\prime 2}}=\|f(x)\| \leq\|x\|+\varepsilon \leq d+\varepsilon, \sqrt{x_{1}^{2}+x_{2}^{2}+x_{3}^{2}} \leq d$ and $0<\varepsilon \leq \frac{1}{13}$. Similarly, it follows from (3.27) that

$$
\left|\left(\left(x_{1}^{\prime}-e_{11}^{\prime}\right)^{2}+x_{2}^{\prime 2}+x_{3}^{\prime 2}\right)-\left(\left(x_{1}-1\right)^{2}+x_{2}^{2}+x_{3}^{2}\right)\right| \leq\left(2 d+\frac{27}{13}\right) \varepsilon,
$$


since $\sqrt{\left(x_{1}^{\prime}-e_{11}^{\prime}\right)^{2}+x_{2}^{\prime 2}+x_{3}^{\prime 2}}=\left\|f(x)-f\left(e_{1}\right)\right\| \leq\left\|x-e_{1}\right\|+\varepsilon \leq d+1+\varepsilon, \sqrt{\left(x_{1}-1\right)^{2}+x_{2}^{2}+x_{3}^{2}}=$ $\left\|x-e_{1}\right\| \leq d+1$ and $0<\varepsilon \leq \frac{1}{13}$. Analogously, by (3.28) and (3.29), we obtain

$$
\left|\left(\left(x_{1}^{\prime}-e_{21}^{\prime}\right)^{2}+\left(x_{2}^{\prime}-e_{22}^{\prime}\right)^{2}+x_{3}^{\prime 2}\right)-\left(x_{1}^{2}+\left(x_{2}-1\right)^{2}+x_{3}^{2}\right)\right| \leq\left(2 d+\frac{27}{13}\right) \varepsilon
$$

and

$$
\left|\left(\left(x_{1}^{\prime}-e_{31}^{\prime}\right)^{2}+\left(x_{2}^{\prime}-e_{32}^{\prime}\right)^{2}+\left(x_{3}^{\prime}-e_{33}^{\prime}\right)^{2}\right)-\left(x_{1}^{2}+x_{2}^{2}+\left(x_{3}-1\right)^{2}\right)\right| \leq\left(2 d+\frac{27}{13}\right) \varepsilon,
$$

since $\sqrt{x_{1}^{2}+\left(x_{2}-1\right)^{2}+x_{3}^{2}} \leq d+1, \sqrt{x_{1}^{2}+x_{2}^{2}+\left(x_{3}-1\right)^{2}} \leq d+1$, and $0<\varepsilon \leq \frac{1}{13}$.

It follows from (3.31) that

$$
\left|\left(x_{1}^{\prime 2}+x_{2}^{\prime 2}+x_{3}^{\prime 2}\right)-\left(x_{1}^{2}+x_{2}^{2}+x_{3}^{2}\right)-2 e_{11}^{\prime} x_{1}^{\prime}+2 x_{1}+e_{11}^{\prime 2}-1\right| \leq\left(2 d+\frac{27}{13}\right) \varepsilon
$$

By using (3.30), we obtain

$$
-\left(4 d+\frac{28}{13}\right) \varepsilon \leq-2 e_{11}^{\prime} x_{1}^{\prime}+2 x_{1}+e_{11}^{\prime 2}-1 \leq\left(4 d+\frac{28}{13}\right) \varepsilon .
$$

Similarly, it follows from (3.32) that

$$
\left|\left(x_{1}^{\prime 2}+x_{2}^{\prime 2}+x_{3}^{\prime 2}\right)-\left(x_{1}^{2}+x_{2}^{2}+x_{3}^{2}\right)-2 e_{21}^{\prime} x_{1}^{\prime}-2 e_{22}^{\prime} x_{2}^{\prime}+2 x_{2}+e_{21}^{\prime 2}+e_{22}^{\prime 2}-1\right| \leq\left(2 d+\frac{27}{13}\right) \varepsilon .
$$

Using (3.30), we obtain

$$
-\left(4 d+\frac{28}{13}\right) \varepsilon \leq-2 e_{21}^{\prime} x_{1}^{\prime}-2 e_{22}^{\prime} x_{2}^{\prime}+2 x_{2}+e_{21}^{\prime 2}+e_{22}^{\prime 2}-1 \leq\left(4 d+\frac{28}{13}\right) \varepsilon .
$$

Analogously, we use (3.30) and (3.33) to obtain

$$
\begin{aligned}
-\left(4 d+\frac{28}{13}\right) \varepsilon & \leq-2 e_{31}^{\prime} x_{1}^{\prime}-2 e_{32}^{\prime} x_{2}^{\prime}-2 e_{33}^{\prime} x_{3}^{\prime}+2 x_{3}+e_{31}^{\prime 2}+e_{32}^{\prime 2}+e_{33}^{\prime 2}-1 \\
& \leq\left(4 d+\frac{28}{13}\right) \varepsilon
\end{aligned}
$$

Moreover, putting $x=e_{1}$ and $y=0$ in (1.3) and using (3.1), we have

$$
\left|e_{11}^{\prime}-1\right| \leq \varepsilon \quad \text { and } \quad\left|e_{11}^{\prime 2}-1\right|=\left|e_{11}^{\prime}-1\right|\left|e_{11}^{\prime}+1\right| \leq \frac{27}{13} \varepsilon .
$$

Therefore, it follows from (3.34) that

$$
\left|x_{1}-x_{1}^{\prime}\right| \leq\left(3 d+\frac{57}{26}\right) \varepsilon
$$

since $\max \left\{\left|x_{1}^{\prime}\right|,\left|x_{2}^{\prime}\right|,\left|x_{3}^{\prime}\right|\right\} \leq\|f(x)\| \leq\|x\|+\varepsilon \leq d+\frac{1}{13}$ and

$$
-2 e_{11}^{\prime} x_{1}^{\prime}+2 x_{1}+e_{11}^{\prime 2}-1=2\left(1-e_{11}^{\prime}\right) x_{1}^{\prime}+2\left(x_{1}-x_{1}^{\prime}\right)+e_{11}^{\prime 2}-1 .
$$


On the other hand, it follows from (3.1) that

$$
\left|2 e_{21}^{\prime} x_{1}^{\prime}\right| \leq 2 c_{21}\left|x_{1}^{\prime}\right| \varepsilon \leq 2 c_{21}\left(d+\frac{1}{13}\right) \varepsilon
$$

By (3.1), we obtain

$$
-\varepsilon \leq 1-e_{22}^{\prime} \leq c_{22} \varepsilon
$$

Furthermore, using (3.35) together with (3.3), we have

$$
-\left(\left(2+c_{21}\right) d+\frac{55+2 c_{21}}{26}\right) \varepsilon \leq x_{2}-e_{22}^{\prime} x_{2}^{\prime} \leq\left(\left(2+c_{21}\right) d+\frac{27+c_{21}}{13}\right) \varepsilon
$$

or

$$
\begin{aligned}
& -\left(\left(2+c_{21}+c_{22}\right) d+\frac{55+2 c_{21}+2 c_{22}}{26}\right) \varepsilon \\
& \quad \leq x_{2}-x_{2}^{\prime} \leq\left(\left(2+c_{21}+c_{22}\right) d+\frac{27+c_{21}+c_{22}}{13}\right) \varepsilon
\end{aligned}
$$

since

$$
x_{2}-e_{22}^{\prime} x_{2}^{\prime}=\left(x_{2}-x_{2}^{\prime}\right)+\left(1-e_{22}^{\prime}\right) x_{2}^{\prime} .
$$

Thus, we see that

$$
\left|x_{2}-x_{2}^{\prime}\right| \leq\left(\left(2+c_{21}+c_{22}\right) d+\frac{55+2 c_{21}+2 c_{22}}{26}\right) \varepsilon
$$

Analogously, by using (3.1), we obtain

$$
\left|2 e_{31}^{\prime} x_{1}^{\prime}\right| \leq 2 c_{31}\left(d+\frac{1}{13}\right) \varepsilon \quad \text { and } \quad\left|2 e_{32}^{\prime} x_{2}^{\prime}\right| \leq 2 c_{32}\left(d+\frac{1}{13}\right) \varepsilon .
$$

Moreover, due to (3.36), we obtain

$$
\begin{aligned}
& -\left(4 d+\frac{28}{13}\right) \varepsilon+2 e_{31}^{\prime} x_{1}^{\prime}+2 e_{32}^{\prime} x_{2}^{\prime}-\left(e_{31}^{\prime 2}+e_{32}^{\prime 2}+e_{33}^{\prime 2}\right)+1 \\
& \quad \leq 2\left(x_{3}-e_{33}^{\prime} x_{3}^{\prime}\right) \leq\left(4 d+\frac{28}{13}\right) \varepsilon+2 e_{31}^{\prime} x_{1}^{\prime}+2 e_{32}^{\prime} x_{2}^{\prime}-\left(e_{31}^{\prime 2}+e_{32}^{\prime 2}+e_{33}^{\prime 2}\right)+1 .
\end{aligned}
$$

In view of (3.19), we have

$$
\begin{aligned}
& -\left(\left(4+2 c_{31}+2 c_{32}\right) d+\frac{55+2 c_{31}+2 c_{32}}{13}\right) \varepsilon \\
& \quad \leq 2\left(x_{3}-e_{33}^{\prime} x_{3}^{\prime}\right) \leq\left(\left(4+2 c_{31}+2 c_{32}\right) d+\frac{54+2 c_{31}+2 c_{32}}{13}\right) \varepsilon
\end{aligned}
$$


By (3.1), we see that $1-c_{33} \varepsilon \leq e_{33}^{\prime} \leq 1+\varepsilon$ and hence,

$$
\left|x_{3}-x_{3}^{\prime}\right| \leq\left(\left(2+c_{31}+c_{32}+c_{33}\right) d+\frac{55+2 c_{31}+2 c_{32}+2 c_{33}}{26}\right) \varepsilon
$$

Finally, we may define an isometry $U: D \rightarrow \mathbb{R}^{3}$ by $U(x)=x=\left(x_{1}, x_{2}, x_{3}\right)$. Then, we use (3.37), (3.38), (3.39), and Lemma 3.3 to obtain

$$
\begin{aligned}
\|f(x)-U(x)\| & =\|f(x)-x\|=\left\|\left(x_{1}^{\prime}-x_{1}, x_{2}^{\prime}-x_{2}, x_{3}^{\prime}-x_{3}\right)\right\| \\
& =\sqrt{\left(x_{1}^{\prime}-x_{1}\right)^{2}+\left(x_{2}^{\prime}-x_{2}\right)^{2}+\left(x_{3}^{\prime}-x_{3}\right)^{2}} \\
& \leq \sqrt{252 d^{2}+134 d+21} \varepsilon \\
& \leq(16 d+5) \varepsilon
\end{aligned}
$$

which completes our proof.

By $c_{i j}$ in Lemma 3.3, (3.37), (3.38), and (3.39), we can express the upper bound of inequality (3.25) more precisely using rational numbers as:

$$
\begin{aligned}
\|f(x)-U(x)\| & =\sqrt{251.5802517 d^{2}+133.1572732 d+20.2971267} \varepsilon \\
& \leq \sqrt{251.5802517 d^{2}+134.9643999 d+18.4900000} \varepsilon \\
& \leq(15.8612816 d+4.3000000) \varepsilon .
\end{aligned}
$$

On the other hand, it follows from [8] that

$$
\begin{aligned}
\|f(x)-U(x)\| & =\sqrt{451 d^{2}+1026 d+587} \varepsilon \\
& \leq \sqrt{451 d^{2}+1029.0519930 d+587} \varepsilon \\
& \leq(21.2367606 d+24.2280829) \varepsilon .
\end{aligned}
$$

Therefore, one can see that the result of this paper is better than that of the previous paper [8].

\section{Discussion}

This paper deals with Fickett's theorem, which can also be called the stability problem of isometries, with a focus on the bounded subsets of $\mathbb{R}^{2}$ or $\mathbb{R}^{3}$.

Fickett provided the authors with a decisive motivation to study this subject. The upper bound introduced in Fickett's theorem for the difference between $\varepsilon$-isometry and exact isometry is unfortunately very large compared to $\varepsilon$ for any sufficiently small $\varepsilon$. This is a big drawback of Fickett's theorem. Thus, the work of further improving Fickett's theorem has to be attractive.

Moreover, the present paper not only proved the local stability of isometries for the case $n=2$, which was not dealt with in the recent paper [8], but also improved the result of [8] when $n=3$.

Finally, it is unclear whether the upper bound for the difference between $\varepsilon$-isometry and exact isometry is independent of the 'radius' $d$ of the domain $D$. It seems unlikely, but it 
would be very surprising if it could be proved that the upper bound is independent of the radius of the domain.

\section{Acknowledgements}

This work was partially supported by the Hallym University Research Fund (HRF-202109-008).

Funding

Hallym University Research Fund (HRF-202109-008).

Availability of data and materials

There are no data that we needed for this manuscript.

\section{Declarations}

\section{Competing interests}

The authors declare that they have no competing interests.

\section{Authors' contributions}

All authors contributed equally to the writing of this paper. All authors read and approved the final manuscript.

\section{Author details}

${ }^{1}$ Mathematics Section, College of Science and Technology, Hongik University, 30016 Sejong, Republic of Korea. ${ }^{2} \|$ song College of Liberal Arts, Hallym University, Chuncheon 24252, Republic of Korea. ${ }^{3}$ School of Materials Science and Engineering, Hongik University, 30016 Sejong, Republic of Korea.

\section{Publisher's Note}

Springer Nature remains neutral with regard to jurisdictional claims in published maps and institutional affiliations.

Received: 7 September 2021 Accepted: 10 January 2022 Published online: 21 January 2022

\section{References}

1. Alestalo, P., Trotsenko, D.A., Väisälä, J.: Isometric approximation. Isr. J. Math. 125, 61-82 (2001)

2. Bhatia, R., Šemrl, P.: Approximate isometries on Euclidean spaces. Am. Math. Mon. 104, 497-504 (1997)

3. Choi, G., Jung, S.-M.: The stability of isometries on restricted domains. Symmetry 13, 282, 11 pages (2021)

4. Fickett, J.W.: Approximate isometries on bounded sets with an application to measure theory. Stud. Math. 72(1), 37-46 (1982)

5. Gruber, P.M.: Stability of isometries. Trans. Am. Math. Soc. 245, 263-277 (1978)

6. Hyers, D.H.: On the stability of the linear functional equation. Proc. Natl. Acad. Sci. USA 27, 222-224 (1941)

7. Hyers, D.H., Ulam, S.M.: On approximate isometries. Bull. Am. Math. Soc. 51, 288-292 (1945)

8. Jung, S.-M.: Hyers-Ulam stability of isometries on bounded domains. Open Math. 19(1), 675-689 (2021)

9. Omladič, M. Šemrl, P.: On non linear perturbations of isometries. Math. Ann. 303, 617-628 (1995)

10. Šemrl, P.: Hyers-Ulam stability of isometries on Banach spaces. Aequ. Math. 58, 157-162 (1999)

11. Sun, L.: Hyers-Ulam stability of $\varepsilon$-isometries between the positive cones of $L^{p}$-spaces. J. Math. Anal. Appl. 487(2), $124014(2020)$

12. Ulam, S.M.: A Collection of Mathematical Problems. Interscience, New York (1960)

13. Väisälä, J.: Isometric approximation property of unbounded sets. Results Math. 43, 359-372 (2003)

\section{Submit your manuscript to a SpringerOpen ${ }^{0}$ journal and benefit from:}

- Convenient online submission

- Rigorous peer review

- Open access: articles freely available online

- High visibility within the field

- Retaining the copyright to your article 